\title{
A possible relation between flare activity in super-luminous supernovae and gamma-ray bursts
}

\author{
Yun-Wei $\mathrm{Yu}^{1,2 \star}$ and Shao-Ze $\mathrm{Li}^{1}$ \\ ${ }^{1}$ Institute of Astrophysics, Central China Normal University, Wuhan 430079, China \\ ${ }^{2}$ Key Laboratory of Quark and Lepton Physics (Central China Normal University), Ministry of Education, Wuhan 430079, China
}

Accepted XXX. Received YYY; in original form ZZZ

\begin{abstract}
Significant undulations appear in the light curve of a recently discovered superluminous supernova (SLSN) SN 2015bn after the first peak, while the underlying profile of the light curve can be well explained by a continuous energy supply from a central engine, possibly the spin-down of a millisecond magnetar. We propose that these undulations are caused by an intermittent pulsed energy supply, indicating an energetic flare activity of the central engine of the SLSN. Many post-burst flares were discovered during X-ray afterglow observations of Gamma-Ray Bursts (GRBs). We find that the SLSN flares described here approximately obey the empirical correlation between the luminosity and time scale of GRB flares, extrapolated to the relevant longer time scales of SLSN flares. This confirms the possible connection between these two different phenomena as previously suggested.
\end{abstract}

Key words: gamma-ray burst: general — stars: neutron — supernovae: specific (SN 2015bn)

\section{INTRODUCTION}

During the past ten years, a series of modern supernova surveys have discovered an unusual type of supernovae with an absolute magnitude at peak emission of $M_{\mathrm{AB}}<-21$, ' which are more luminous than normal supernovae by a factor of $\sim 10-100$ (Benetti et al. 2014; Bersten et al. 2016; ' Chatzopoulos et al. 2011; Chen et al. 2016a; Chomiuk et al. 2011; Chornock et al. 2013; Gal-Yam et al. 2009, 2012; Howell et al. 2013; Inserra et al. 2013, 2016; Kangas et al. 2016; Leloudas et al. 2012; Lunnan et al. 2013, 2016; McCrum et al. 2014, 2015; Nicholl et al. 2013, 2016; Ofek et al. 2007; Papadopoulos et al. 2015; Quimby et al. 2007, 2011; Smith et al. 2007, 2016; Vreeswijk et al. 2014; Yan et al. 2016).

The total radiated energy of a typical superluminous supernova (SLSN) is on the order of $\sim 10^{51} \mathrm{erg}$. If this radiation is mainly powered as usual by the radioactive chain ${ }^{56} \mathrm{Ni} \rightarrow{ }^{56} \mathrm{Co} \rightarrow{ }^{56} \mathrm{Fe}$, then an extremely large amount (several to several tens of solar masses) of radioactive ${ }^{56} \mathrm{Ni}$ would be required. In principle, such a high mass of ${ }^{56} \mathrm{Ni}$ could be produced by core-collapse explosions of very massive progenitors with a very large explosion energy (Umeda \& Nomoto 2008; Moriya et al. 2010) or by disruption explosions of very massive progenitors due to pair-production instability (Barkat et al. 1967; Heger \& Woosley 2002; Gal-Yam

\footnotetext{
^ E-mail: yuyw@mail.ccnu.edu.cn
}

et al. 2009). In both cases, however, the corresponding high masses of supernova ejecta would lead to a broad slowlyevolving supernova light curve, whereas the observational light curves rise and often decline rapidly. Moreover, for the pair-instability events, there is still controversy whether they occur locally and if they are related to some SLSNe or not (e.g. McCrum et al. 2014; Georgy et al. 2017). Therefore, generally speaking, the radioactivity power scenario is seriously challenged by the very high luminosity of SLSNe.

Alternatively, a powerful central engine is believed to play an essential role in driving SLSN explosions and in powering their emission, by an instantaneous and/or a longlasting energy injection into the explosion-ejected stellar envelope. To be specific, at the initial time of some SLSN explosions, their central engines could impulsively provide a great amount of energy to the supernova ejecta, and lead the ejecta to have a very high initial velocity corresponding to a kinetic energy on the order of $10^{52} \mathrm{erg}$. If these explosions happen in dense, extended circum-stellar material (CSM; e.g., stellar wind and some particular material clusters), then the ejecta can be subsequently heated by the conversion of the kinetic energy through shock interaction between the ejecta and surrounding material (Smith \& McCray 2007; Chevalier \& Irwin 2011; Moriya et al. 2011, 2013; Ginzburg \& Balberg 2012; Inserra et al. 2016). Such interaction-powered supernovae can usually be indicated by narrow Balmer emission lines (Chatzopoulos et al. 2011). 
On the contrary, for hydrogen-poor SLSNe, shock interaction could usually be negligible, and the supernova emission is probably powered directly by a long-lasting central engine. During the past few years, a remarkable number of light curves of SLSNe, even including several hydrogen-rich ones without narrow lines, have been successfully explained with a continuous energy injection (Dessart et al. 2012; Inserra et al. 2013, 2016; Nicholl et al. 2013, 2016; Howell et al. 2013; McCrum et al. 2014; Wang et al. 2015; Dai et al. 2016; Lunnan et al. 2016; Bersten et al. 2016; Yu et al. 2017). This indicates that the central engine could be the most viable and most common energy source for most SLSNe, which makes these SLSNe very relevant to another engine-driven phenomenon: gamma-ray bursts (GRBs). In principle, different energy sources could coexist in some SLSNe (e.g. Wang et al. 2015a; Lunnan et al. 2016).

In the framework of the long-lasting energy injection model, it is convenient to connect SLSN light curves with the temporal behaviors of their central engines. Specifically, while a continuous energy injection determines the basic profile of the SLSN light curves, it can also be expected that some light curve undulations could be caused by flare activity of the central engines. In this case, the modeling of these light curve undulations could provide a special insight in probing the nature of these SLSN engines and even their possible connection with GRB engines.

\section{SN 2015BN}

Recently, Nicholl et al. (2016) presented the multiwavelength observational results of Type I SN 2015bn, where some significant undulations appear in its light curve during the first $\sim 150$ days. The dataset they provided is extensive and detailed, which enables us to constrain and even distinguish different SLSN models. As investigated in Nicholl et al. (2016), the basic profile of the bolometric light curve of SN 2015bn, excluding the undulation components, could in principle be modeled by a scenario of that the fast evolving peak emission is caused by ejecta-CSM interaction, while the late-time slowly-decline emission is dominated by ${ }^{56} \mathrm{Co}$ decays. However, it could still be very difficult (if not impossible) to simultaneously model the light curve undulations by successive collisions of the supernova ejecta with some massive shells that were expelled prior to the supernova explosion, where the structure of progenitor and its mass-loss history must be designed and tuned elaborately. Another possibility they discussed is that this SLSN is powered as usual by a long-lasting central engine, which is on the focus of this paper. In our opinion, while the underlying smooth light curve is constructed by a continuous energy injection, the light curve undulations of SN 2015bn are probably associated with the late flare activity of its central engine.

\section{FITTINGS AND RESULTS}

\subsection{Basic equations}

Following Kasen \& Bildsten (2010), the evolution of the internal energy $E_{\text {int }}$ of a supernova ejecta can be determined by (Yu et al. 2015)

$\frac{d E_{\text {int }}}{d t}=L_{\text {in }}-L_{\mathrm{sn}}-4 \pi R^{2} v p$,

where $t$ is the time, $L_{\mathrm{in}}$ is the energy injection rate, $L_{\mathrm{sn}}$ is the supernova luminosity, $R$ and $v=d R / d t$ are the radius and speed of supernova ejecta, and $p$ is the pressure that can be related to the internal energy by $p=\frac{1}{3}\left(E_{\text {int }} / \frac{4}{3} \pi R^{3}\right)$. The dynamical evolution of the supernova ejecta is given by $d v / d t=4 \pi R^{2} p / M_{\mathrm{ej}}$, where $M_{\mathrm{ej}}$ is the total mass of ejecta. Then, by considering of the heat diffusion in the supernova ejecta (see Eq. 11 in Kasen \& Bildsten 2010), the bolometric luminosity of the supernova can be roughly determined by the following formula:

$L_{\mathrm{sn}}=\frac{c E_{\text {int }}}{R \tau}\left(1-e^{-\tau}\right)$,

where $c$ is the speed of light, $\tau=3 \kappa M_{\mathrm{ej}} / 4 \pi R^{2}$ is the optical depth, and $\kappa$ is the opacity. For $\tau \gg 1$ the above equation reads $L_{\mathrm{sn}}=c E_{\mathrm{int}} /(R \tau)$, while $L_{\mathrm{sn}}=c E_{\mathrm{int}} / R$ for $\tau \ll 1$ (e.g., see Kotera et al. 2013).

\subsection{The underlying profile of light curve}

Continuous energy release from a SLSN engine could be due to the spin-down of a millisecond magnetar (Woosley et al. 2010; Kasen et al. 2010) or due to the feedback of fallback accretion onto a magnetar or a black hole (Dexter \& Kasen 2013). In literature, the former model was employed more widely than the latter. The energy injection rate in the spinning-down magnetar model can be expressed by the spin-down luminosity of the magnetar as

$L_{\text {in }}(t)=L_{\text {in }}(0)\left(1+t / t_{\mathrm{sd}}\right)^{-2}$,

where the initial luminosity $L_{\mathrm{in}}(0)$ and the spin-down timescale $t_{\mathrm{sd}}$ are taken as free parameters when we interpret the basic trends of the light curve. As shown in Figure 1 (see also Figure 19 in Nicholl et al. 2016), the light curve of SN 2015bn can well be profiled by the magnetar engine model. The excess of the first data could be caused by the magnetardriven shock breakout emission (Kasen et al. 2016), while the drop of the last three data is due to the leakage of highenergy photons after the ejecta gradually becomes transparent (Wang et al. 2015). By introducing the expressions of the initial spin-down luminosity $L_{\mathrm{in}}(0)=10^{7} B_{p}^{2} P(0)^{-4} \mathrm{erg} \mathrm{s}^{-1}$ and the timescale $t_{\mathrm{sd}}=2 \times 10^{39} B_{p}^{-2} P(0)^{2} \mathrm{~s}$, we can derive the dipolar magnetic field strength and the initial spin period of the magnetar to be $B_{p}=6.4 \times 10^{13} \mathrm{G}$ and $P(0)=2.3$ $\mathrm{ms}$. The parameter values obtained here are somewhat different from those presented in Yu et al. (2017), because the detailed structure of the light curve was not taken into account there.

For a comparison, we also consider that the continuous energy injection comes from an outflow that is driven as the feedback (e.g. disk wind) of a fallback accretion onto the central compact object (a magnetar or a black hole). By assuming a direct proportion to the fallback accretion rate, the outflow luminosity due to the accretion feedback can be written as (Piro \& Ott 2011)

$L_{\text {in }}(t)=L_{\text {in }}(0)\left[\left(t / t_{\text {accr }}\right)^{-1 / 2}+\left(t / t_{\text {accr }}\right)^{5 / 3}\right]^{-1}$.

Here the accretion timescale $t_{\text {accr }}$ can roughly be estimated 
by a free-fall timescale of $\sim\left(G \rho_{0}\right)^{-1 / 2}$ (Dexter \& Kasen 2013), which gives a typical value of a few hundred to a thousand of seconds for a typical stellar density of $\rho_{0} \sim$ $300 \mathrm{~g} \mathrm{~cm}^{-3}$. During such a short time, most of extractable energy of the fallback material has been released and injected into the supernova ejecta. This quickly injected energy would mostly be converted into the kinetic energy of the ejecta, which leads to a high velocity of about $3 \times 10^{9} \mathrm{~cm} \mathrm{~s}^{-1}$. This velocity is much higher than the observed photosphere velocity of SN $2015 \mathrm{bn}$ of $\sim 9 \times 10^{8} \mathrm{~cm} \mathrm{~s}^{-1}$ (Nicholl et al. 2016), which somewhat disfavors the accretion feedback model, although a precise calculation of photosphere velocity is dependent on the specific matter distributions in the ejecta. Meanwhile, the supernova light curve is predicted to decrease too quickly to be consistent with the data after the peak, if an ejecta mass of $16 M_{\odot}\left(\right.$ for $\left.\kappa=0.1 \mathrm{~cm}^{2} \mathrm{~g}^{-1}\right)$ is taken properly to fit the peak time. As an attempt to save this model, we tentatively introduce an artificial mass of $8 M_{\odot}$ of radioactive ${ }^{56} \mathrm{Ni}$ to improve the modeling of the late emission, as shown in Figure 2. In principle, this putative nickel mass could be produced by core-collapse explosion 1 of $\mathrm{a} \sim 100 M_{\odot}$ progenitor (Umeda \& Nomoto 2008), if the explosion energy can reach $>5 \times 10^{52} \mathrm{erg}$, which could be provided by the accretion feedback. However, the extremely high mass of the progenitor would still lead to a too heavy supernova ejecta, the mass of which is at least higher than the $\mathrm{CO}$ core of the star of $\sim 40 M_{\odot}$ even if the hydrogen and helium envelopes have been lost completely. This mass is too high to be consistent with the ejecta mass of $16 M_{\odot}$ inferred from the fitting.

Therefore, in comparison, the spinning-down magnetar model can explain the underlying profile of the light curve of SN 2015bn more naturally and more self-consistently than the accretion feedback model. In the following calculations, we will only take the magnetar model into account.

\subsection{Light curve undulations and flare activity}

A long-lasting central engine actually has been widely suggested to account for many features of afterglow emission of GRBs. On one hand, continuous energy release, usually from a spinning-down millisecond magnetar, was always employed to explain the shallow-decay or plateau afterglows of GRBs (Dai \& Lu 1998a,b; Zhang \& Meszaros 2001; Yu et al. 2010; Rowlinson et al. 2013; Lü et al. 2015). On the other hand, more interestingly, a great number of rapidly rising and declining X-ray flares have been widely discovered in about one-third of Swift GRBs (Burrows et al. 2005; Falcone et al. 2007; Chincarini et al. 2007; Wang \& Dai 2013; Yi et al. 2016). This robustly indicates that many intermittent and energetic activities have taken place on the GRB engines. By considering of the high relevance between GRBs and SLSNe (Yu et al. 2017), it is natural to consider that the central engines of SLSNe may also be able to make flare activity. As a result, some undulations like those appearing in

1 The pair-instability supernova model can not be employed here because of the pre-assumed presence of the central compact object. In any case, this model would also predict a too high ejecta mass.

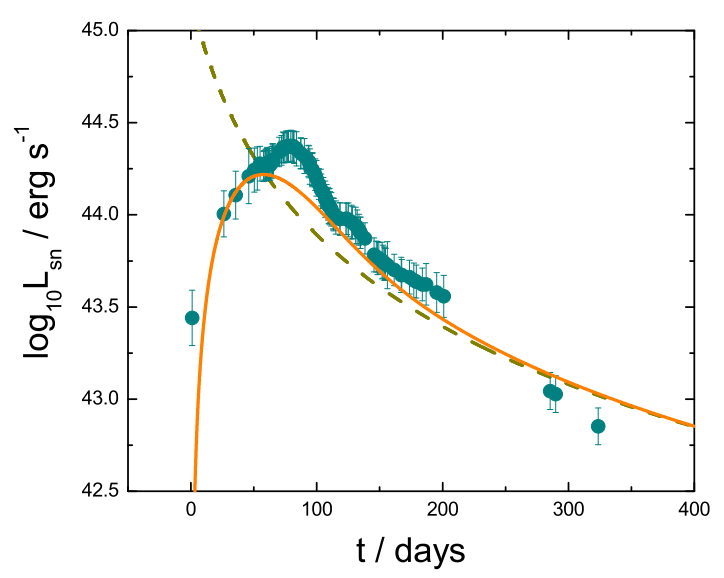

Figure 1. A fitting to the underlying profile of light curve of SN 2015b in the spinning-down magnetar model with parameters $L_{\text {in }}(0)=1.5 \times 10^{45} \mathrm{erg} \mathrm{s}^{-1}$ and $t_{\mathrm{sd}}=30$ day (solid line). The dashed line represents the energy injection rate. The parameters for the supernova ejecta are adopted to $\kappa=0.1 \mathrm{~cm}^{2} \mathrm{~g}^{-1}, M_{\mathrm{ej}}=$ $10 M_{\odot}$, and initial velocity $v(0)=7.5 \times 10^{8} \mathrm{~cm} \mathrm{~s}^{-1}$.

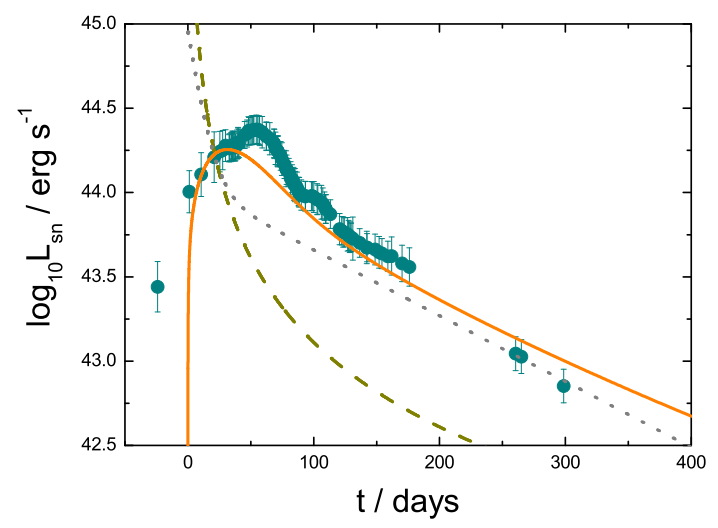

Figure 2. A tentative fitting to the underlying profile of light curve of SN 2015b by combining the heating effects due to accretion feedback and radioactive decays of ${ }^{56} \mathrm{Ni}$ (Solid line). The energy injection rates due to accretion feedback and radioactivity are presented by the dashed and dotted lines, respectively. The model parameters are taken as $L_{\text {in }}(0)=4.7 \times 10^{49} \mathrm{erg} \mathrm{s}^{-1}$, $t_{\text {accr }}=1000 \mathrm{~s}, \kappa=0.1 \mathrm{~cm}^{2} \mathrm{~g}^{-1}, M=16 M_{\odot}, v(0)=5 \times 10^{8} \mathrm{~cm} \mathrm{~s}^{-1}$ and $M_{\mathrm{Ni}}=8 M_{\odot}$.

the light curve of SN 2015bn could be caused by the delayed flaring energy release.

For an empirical fitting to the light curve of SN 2015bn, we describe the energy release rate due to an engine flare by the following formula:

$L_{\text {flare }}(t)=L_{\text {flare }, \mathrm{p}}\left[\left(\frac{t}{t_{\text {flare }, \mathrm{p}}}\right)^{\alpha_{1} w}+\left(\frac{t}{t_{\text {flare }, \mathrm{p}}}\right)^{\alpha_{2} w}\right]^{-1 / w}$

which was usually adopted to fit the light curves of GRB $\mathrm{X}$-ray flares, where the structure parameters $\alpha_{1}, \alpha_{2}$, and $w$ reflect the sharpness and smoothness of the flares. By assuming a possible universal nature of flare activity, we take 
Table 1. Flare Parameters

\begin{tabular}{cc}
\hline \hline$L_{\text {flare }, \mathrm{p}} / 10^{43} \mathrm{erg} \mathrm{s}^{-1}$ & $t_{\text {flare } \mathrm{p}} /$ day \\
\hline 32 & 76 \\
5.5 & 123 \\
1.5 & 180 \\
\hline \hline
\end{tabular}

values of the structure parameters refering to the fitting results of GRB flares (Yi et al. 2016). Nevertheless, different from the GRB situation, here we cannot directly confront Equation (5) with the supernova light curve, because the flare energy can be completely absorbed by the optical thick supernova ejecta. In other words, engine flares can influence the supernova emission as pulsing energy injections. Therefore, the consequent supernova light curves are primarily related to the parameters $L_{\text {flare,p }}$ and $t_{\text {flare,p }}$, but insensitive to the structure parameters. By substituting expression (5) into Equation (11), we attempt to model the light curve undulations of SN 2015bn by engine flare activity. Consequently, a perfect result is shown in Figure 3, where three flares are invoked, which empirically demonstrates the availability of the flare explanation of the light curve undulations.

The parameters of the three SLSN flares are listed in Table 1. We also plot these flares in the $L_{\text {flare,p }}-t_{\text {flare,p }}$ plane in Figure 4, where 200 GRB X-ray flares are presented for a comparison. On one hand, for these GRB flares, an apparent correlation of $L_{\text {flare,p }} \propto t_{\text {flare,p }}^{-1.27}$ was found by Yi et al. (2016), although a large uncertainty of about two orders of magnitude exists. On the other hand, as shown in Figure 4 , the SLSN flares have much longer timescales and lower luminosity than the GRB flares. In despite of these differences, we still find that the three SLSN flares can basically fall into the $2-\sigma$ uncertain region of the extension of the GRB flare correlation. Such a consistency indicates that the central engines of SLSNe and GRBs have a common behavior characteristics, which could be a natural result of their same magnetar nature although the magnetic field strengths of them are very different (Yu et al. 2017).

The statistics of GRB flares shows that the engines are usually more active at earlier time. Then, one may query that why we can only detect three very late flares at this first time and why we have never yet detected any signature for more frequent earlier flares. Our answer to these questions is, even though the temporal evolution of flares is very sharp, the undulations they can make in supernova light curves are usually gentle, because of the trapping of photons in the ejecta before the ejecta becomes completely transparent. The earlier the time, the more serious the trapping effect. As a result, any fluctuation in the energy release process of an engine can in principle be smoothed in the consequent light curve, in particular, before the photon diffusion timescale. In other words, it is nearly impossible to detect any obvious signature of early flares from a supernova light curve before its peak, no matter how many flares have happened then. Nevertheless, in view of the relatively lower magnetic fields of SLSN magnetars than GRB magnetars (Yu et al. 2017), it

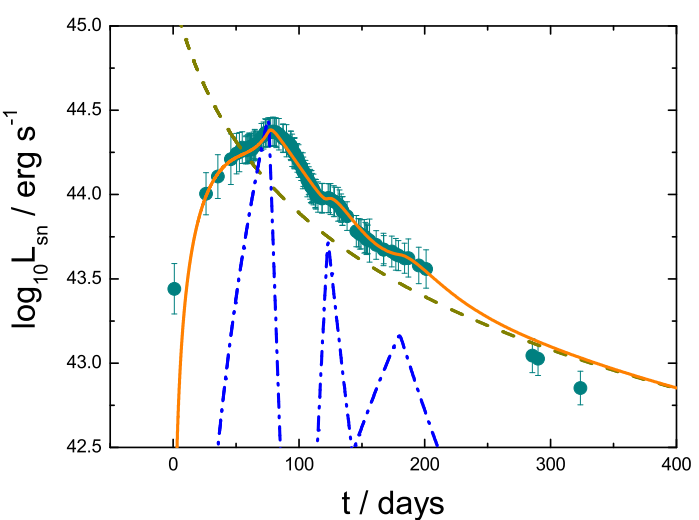

Figure 3. Fittings to the light curve of SN 2015bn (solid lines) in the spinning-down magnetar model. For modeling the undulations, three flaring intermittent energy injections are invoked (dash-dotted lines). The parameters for the continuous energy injections are the same to Figure 1.

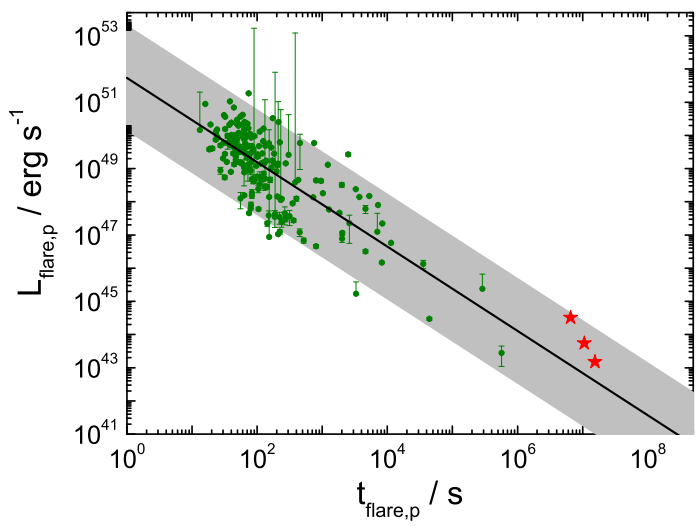

Figure 4. A comparison of the flares of SN 2015bn (stars) with 200 GRB flares (solid circles) in the peak luminosity-peak time plane. The GRB data is taken from Yi et al. (2016). The solid line presents the correlation between the peak luminosities and peak times of these GRB flares and the shaded region represents the $2-\sigma$ uncertain region of the relation.

is still reasonable to consider that the start time of the flare activity of SLSNe is intrinsically later than that of GRBs.

\section{CONCLUSION AND DISCUSSIONS}

Recently, it has been suggested that Type I SLSNe and long GRBs could be two branches from an united origin (Metzger et al. 2015; Yu et al. 2017). To be specific, both are produced as outcomes of the formation of a millisecond magnetar from core-collapse of a massive rapidly rotating progenitor star. The primary difference between them is the magnetic field strengths of their magnetars, which leads to various observational differences between these two explosion phenomena. The discovery of the light curve undulations of SN 2015bn, which is very fortunate, indicates that 
late but energetic flare activity has taken place on its central engine. This strongly reveals the similarity and connection between SLSNe and GRBs from a new perspective, in view of the ubiquitous existence of GRB flares. The possible universal $L_{\text {flare,p }}-t_{\text {flare,p }}$ relationship further indicates that the central engines of SLSNe and GRBs could have a common nature, e.g., the magnetar nature as supposed. This provides an independent and robust support to the suggestion of Metzger et al. (2015) and Yu et al. (2017). The different energy and time scales of the flares of SLSNe and GRBs could arise from the different magnetic fields of their magnetar engines.

In the framework of the magnetar engine model, we tend to believe that the flare activity is associated with reconnections of ultra-high multi-polar magnetic fields in the magnetar, by referring to the magnetic reconnection model previously proposed by Dai et al. (2006) for explaining GRB flares. Although the dipolar field derived above for SN 2015bn is not so high, the discovery of some so-called low-field magnetars in our galaxy (e.g. Rea et al. 2010) indicates that the internal multi-polar fields of a magnetar could be much higher than its surface dipolar field. In Dai et al. (2006), a strong internal toroidal field is considered to form due to differential rotation of the magnetar and subsequently float to the stellar surface to be reconnected. Qualitatively, this scenario could have many similarities with the physical processes of solar flares. This was statistically confirmed by Wang \& Dai (2013), who discovered that the distributions of energies, durations, and waiting times of both GRB flares and solar flares can all be understood within the physical framework of self-organizedcriticality avalanche-like processes. Nevertheless, in Dai et al. (2006), the magnetar is assumed to simply consist of a clearly-separated solid crust and core, which is probably invalid for an extremely hot magnetar. An actual evolution of magnetic fields of a newly-born magnetar could be much more complicated than that considered in Dai et al. (2006). For example, fluid instabilities could be involved (e.g. Cheng $\& \mathrm{Yu}$ 2014). Therefore, an elaborate model is demanded to quantitatively describe the magnetic reconnections and to account for the properties of the observed SLSN flares as well as the related GRB flares.

An elaborate model is also needed to consolidate our flare explanation for the light curve undulations and distinguishing it from other possible scenarios (e.g., the CSM interaction model). First of all, the dynamics of the supernova ejecta and the radiation transfer in it need to be described by a more detailed radiation hydrodynamic code such as the public SuperNova Explosion Code (SNEC; Morozova et al. 2015). Furthermore, in contrast to the 1D case considered here, a 2D simulation of Chen et al. (2016) showed that the interaction between a magnetar wind and a supernova ejecta can in fact lead to many fluid instabilities, which mix the ejecta material and fracture the ejecta into filamentary structure. The consequent inhomogenity and anisotropy of the system could substantially influence the early dynamics and emission of the supernova. It can even be expected that the clumpy structure of the ejecta could also cause some light curve undulations, which is worth to be investigated in future simulations. In any case, it will be crucial and helpful to collect more observational data to exhibit the details of early light curves (in particular during the increasing phase) and to implement some synergic multi-wavelength observations to SLSNe including in the high-energy bands.

\section{ACKNOWLEDGEMENTS}

The Authors thank M. Nicholl and F. Y. Wang for sharing the data of SN 2015bn and GRB flares, respectively. This work is supported by the National Natural Science Foundation of China (grant No. 11473008) and the Program for New Century Excellent Talents in University (grant No. NCET13-0822).

\section{REFERENCES}

Barkat Z., Rakavy G., Sack N., 1967, PhRvL, 18, 379

Benetti S., et al., 2014, MNRAS, 441, 289

Bersten M. C., Benvenuto O. G., Orellana M., Nomoto K., 2016, ApJ, 817, L8

Burrows D. N., et al., 2005, Sci, 309, 1833

Cano Z., Johansson Andreas K. G., Maeda K., 2016, MNRAS, 457, 2761

Chatzopoulos E., et al., 2011, ApJ, 729, 143

Chen K.-J., Woosley S. E., Sukhbold T., 2016, ApJ, 832, 73

Chen T.-W., et al., 2016, arXiv, arXiv:1611.09910

Cheng Q., Yu Y.-W., 2014, ApJ, 786, L13

Chevalier R. A., Irwin C. M., 2011, ApJ, 729, L6

Chincarini G., et al., 2007, ApJ, 671, 1903

Chomiuk L., et al., 2011, ApJ, 743, 114

Chornock R., et al., 2013, ApJ, 767, 162

Dai Z. G., 2004, ApJ, 606, 1000

Dai Z. G., Lu T., 1998, PhRvL, 81, 4301

Dai Z. G., Lu T., 1998, A\&A, 333, L87

Dai Z. G., Wang S. Q., Wang J. S., Wang L. J., Yu Y. W., 2016, ApJ, 817, 132

Dai Z. G., Wang X. Y., Wu X. F., Zhang B., 2006, Sci, 311, 1127

Dessart L., Hillier D. J., Waldman R., Livne E., Blondin S., 2012, MNRAS, 426, L76

Dexter J., Kasen D., 2013, ApJ, 772, 30

Dong S., et al., 2016, Sci, 351, 257

Falcone A. D., et al., 2007, ApJ, 671, 1921

Gal-Yam A., et al., 2009, Natur, 462, 624

Gal-Yam A., 2012, Sci, 337, 927

Gao H., Lei W.-H., You Z.-Q., Xie W., 2016, ApJ, 826, 141

Ginzburg S., Balberg S., 2012, ApJ, 757, 178

Greiner J., et al., 2015, Natur, 523, 189

Heger A., Woosley S. E., 2002, ApJ, 567, 532

Howell D. A., et al., 2013, ApJ, 779, 98

Inserra C., et al., 2016, arXiv, arXiv:1604.01226

Inserra C., et al., 2013, ApJ, 770, 128

Kangas T., et al., 2016, arXiv, arXiv:1611.10207

Kann D. A., et al., 2016, arXiv, arXiv:1606.06791

Kasen D., Bildsten L., 2010, ApJ, 717, 245

Kasen D., Metzger B. D., Bildsten L., 2016, ApJ, 821, 36

Kotera K., Phinney E. S., Olinto A. V., 2013, MNRAS, 432, 3228

Lü H.-J., Zhang B., Lei W.-H., Li Y., Lasky P. D., 2015, ApJ, 805,89

Leloudas G., et al., 2012, A\&A, 541, A129

Lunnan R., et al., 2013, ApJ, 771, 97

Lunnan R., et al., 2016, ApJ, 831, 144

McCrum M., et al., 2014, MNRAS, 437, 656

McCrum M., et al., 2015, MNRAS, 448, 1206

Metzger B. D., Margalit B., Kasen D., Quataert E., 2015, MNRAS, 454, 3311

Morozova V., Piro A. L., Renzo M., Ott C. D., Clausen D., Couch S. M., Ellis J., Roberts L. F., 2015, ApJ, 814, 63 
Nicholl M., et al., 2016, ApJ, 826, 39

Nicholl M., et al., 2013, Natur, 502, 346

Ofek E. O., et al., 2007, ApJ, 659, L13

Papadopoulos A., et al., 2015, MNRAS, 449, 1215

Quimby R. M., et al., 2011, Natur, 474, 487

Quimby R. M., Aldering G., Wheeler J. C., Höflich P., Akerlof C. W., Rykoff E. S., 2007, ApJ, 668, L99

Rea N., et al., 2010, Sci, 330, 944

Rowlinson A., O'Brien P. T., Metzger B. D., Tanvir N. R., Levan A. J., 2013, MNRAS, 430, 1061

Smith M., et al., 2016, ApJ, 818, L8

Smith N., et al., 2007, ApJ, 666, 1116

Umeda H., Nomoto K., 2008, ApJ, 673, 1014-1022

Vreeswijk P. M., et al., 2014, ApJ, 797, 24

Wang F. Y., Dai Z. G., 2013, NatPh, 9, 465

Wang S. Q., Liu L. D., Dai Z. G., Wang L. J., Wu X. F., 2016, ApJ, 828, 87

Wang S. Q., Wang L. J., Dai Z. G., Wu X. F., 2015, ApJ, 799, 107

Woosley S. E., 2010, ApJ, 719, L204

Woosley S. E., Blinnikov S., Heger A., 2007, Natur, 450, 390

Yan L., et al., 2016, arXiv, arXiv:1611.02782

Yi S.-X., Xi S.-Q., Yu H., Wang F. Y., Mu H.-J., Lü L.-Z., Liang E.-W., 2016, ApJS, 224, 20

Yu Y.-W., Cheng K. S., Cao X.-F., 2010, ApJ, 715, 477

Yu Y.-W., Li S.-Z., Dai Z.-G., 2015, ApJ, 806, L6

Yu Y.-W., Zhu J.-P., Li S.-Z., Lü H.-J., Zou Y.-C., 2017, arXiv, arXiv:1704.01682

Zhang B., Mészáros P., 2001, ApJ, 552, L35 